\title{
Distribution of time to buffer overflow in a finite- buffer manufacturing model with unreliable machine
}

\author{
Wojciech M. Kempa ${ }^{1, *}$, Iwona Paprocka ${ }^{2}$, Cezary Grabowik ${ }^{2}$, Krzysztof Kalinowski ${ }^{2}$, and \\ Damian Krenczyk ${ }^{2}$ \\ ${ }^{1}$ Silesian University of Technology, Faculty of Applied Mathematics, Institute of Mathematics, 23 \\ Kaszubska Str., 44-100 Gliwice, Poland \\ ${ }^{2}$ Silesian University of Technology, Faculty of Mechanical Engineering, Institute of Engineering \\ Processes Automation and Integrated Manufacturing Systems, 18A Konarskiego Str., 44-100 Gliwice, \\ Poland
}

\begin{abstract}
One of the most important characteristic of each queueing model is time to buffer overflow. In the paper the distribution of that time in a single-channel manufacturing system, modelled by a Markovian finitebuffer queue with machine breakdowns, is studied. By using the analytical approach based on the idea of embedded Markov chain, total probability law and linear algebra, the formula for Laplace transform of the time to buffer overflow conditional distribution is found. Numerical illustration is presented.
\end{abstract}

\section{Preliminaries}

The problem of finite buffer (magazine) capacity is a typical one in manufacturing engineering. Jobs arriving to the system being saturated are being lost or must be redirected to another destination. The in-depth investigation of the loss process cannot be based only on the well-known coefficient as the loss ratio, defined as the part of the total number of jobs being directed to the system (manufacturing line), which are lost due to the buffer overflow. To analyze the phenomenon more precisely from the probabilistic point of view, it is necessary to know, e.g. the probability distributions of buffer overflow durations and times of reaching them. The problem becomes more essential in the case of unreliable machine, where successive failure-free times are followed by repair periods during which the processing of jobs is suspended.

In [1] and [2] the studies on the application of different-type queueing models in manufacturing engineering can be found. As one may notice, the analytical results for distributions of the time to the buffer overflow in one-channel queues modelling singlemachine manufacturing lines, in fact, are restricted mainly to systems without machine breakdowns. In [3-4] the formulae for the time-to- buffer overflow period were obtained for

\footnotetext{
${ }^{*}$ Corresponding author: wojciech.kempa@polsl.pl
} 
models with BMAP (Batch Markovian Arrival Process) and MMPP (Markov-Modulated Poisson Process) input flows, respectively. One can find another results related to buffer overflow period and the loss process, e.g. in [5] and [6]. Compact-form representation for the LT (Laplace transform) of time-to-buffer overflow distribution for the model with Poisson arrival stream and setup/closedown times was derived in [7]. In [8] (see also [9]) a similar formula was obtained for the GI/M/1/N-type queueing system (using the wellknown Kendall's notation of queueing systems).

In the paper the conditional CDF (cumulative distribution function) tail of the time $\beta$ to the buffer overflow, conditioned by the number $X(0)$ of jobs present in the system at the opening, is studied, i.e. the stochastic characteristic

$$
B_{n}(t) \stackrel{\text { def }}{=} \boldsymbol{P}\{\beta>t \mid X(0)=n\}, \quad t>0, \quad 0 \leq n \leq N-1,
$$

where $N$ stands for the system capacity. By applying the approach based on the memoryless property of exponential distribution and the formula of total probability, a system of integral equations for the conditional time-to-buffer overflow distribution is derived. The solution of the corresponding system written for LTs is obtained in a compact form using the linear-algebraic approach.

\section{Model description}

In the paper a model of a single-machine manufacturing line described by a finite-buffer one-channel $\mathrm{M} / \mathrm{M} / 1 / \mathrm{N}$-type queueing system is investigated, in which the input flow of jobs is described by a Poisson process with rate $\lambda$, and the processing of a single job is provided in an exponential service time with mean $\mu^{-1}$, according to the FIFO (First In First Out) service discipline. In the case if an arriving job finds the machine busy with processing, it joins the finite-capacity buffer (magazine) queue and waits for service. The maximum number of jobs present simultaneously in the system is bounded by $N$, i.e. we have $N-1$ places in the buffer and one place in the processing machine. If the arriving job finds the system being saturated, i.e. with $N$ jobs present, then this job is lost.

The system starts the evolution with a machine being functional at time $t=0$. The working (failure-free) period is assumed to be exponentially distributed with mean $\gamma^{-1}$. At the completion moment of the failure-free period a machine breakdown occurs and a generally distributed repairing time with $\operatorname{CDF} G(\cdot)$ begins. After finishing the repair time, a new failure-free period begins, and so on. The sequences of random variables representing successive inter-arrival, processing, failure-free and repairing times are supposed to be totally independent. Additionally, it is assumed that the magazine (buffer) can contain some jobs before the start of the processing (before time $t=0$ ).

\section{Integral equations for time-to-buffer overflow distribution}

Let us start with considering the case when the magazine is empty before the starting moment of the system.

Considering the situations in which the first job arrives before and after time $t$, we obtain:

$$
B_{0}(t)=\lambda \int_{0}^{t} e^{-\lambda x} B_{1}(t-x) d x+e^{-\lambda t} .
$$

In the case if at least one job is accumulated in the buffer queue before the opening of the system, the probability distribution of the time to buffer overflow depends on times of occurrences of the first job arrival, first job departure and first machine breakdown. 
Indeed, let us define the following random events:

- $\quad D_{1}$ - before time epoch $t$ the first job arrives and precedes the first departure epoch and the first machine breakdown;

- $\quad D_{2}$ - before time $t$ the first job departure occurs, and precedes the first arrival epoch and the first machine breakdown;

- $\quad D_{3}$ - before time $t$ the first machine breakdown occurs, and precedes the first arrival and departure epochs;

- $\quad D_{4}$ - before time $t$ neither the first job arrival, nor job departure, nor the first machine breakdown occurs.

Due to the fact that for a fixed time $t$ random events $D_{i}$, for $i=1,2,3,4$, are separable in pairs and $\boldsymbol{P}\left\{D_{1}\right\}+\boldsymbol{P}\left\{D_{2}\right\}+\boldsymbol{P}\left\{D_{3}\right\}+\boldsymbol{P}\left\{D_{3}\right\}=1$, we can then write:

$$
B_{n}(t)=\sum_{i=1}^{4} B_{n}^{(i)}(t) \stackrel{\text { def }}{=} \sum_{i=1}^{4} \boldsymbol{P}\left\{(\beta>t) \cap D_{i} \mid X(0)=n\right\},
$$

where $t>0$ and $1 \leq n \leq N-1$.

Suppose now that the buffer (magazine) is not empty at the starting time, respectively i.e. $1 \leq n \leq N-1$. By applying the continuous version of the total probability law and by using the memoryless property of the exponential distribution, we get the following representations:

$$
\begin{gathered}
B_{n}^{(1)}(t)=\left(1-\delta_{n, N-1}\right) \lambda \int_{0}^{t} e^{-(\lambda+\mu+\gamma) x} B_{n+1}(t-x) d x, \\
B_{n}^{(2)}(t)=\mu \int_{0}^{t} e^{-(\lambda+\mu+\gamma) x} B_{n-1}(t-x) d x, \\
B_{n}^{(3)}(t)=\gamma \int_{x=0}^{t} e^{-(\lambda+\mu+\gamma) x} d x\left[\int_{y=0}^{t-x} \sum_{k=0}^{N-n-1} \frac{(\lambda y)^{k}}{k !} e^{-\lambda y} B_{n+k}(t-x-y) d G(y)\right. \\
\left.+\bar{G}(t-x) \sum_{k=0}^{N-n-1} \frac{[\lambda(t-x)]^{k}}{k !} e^{-\lambda(t-x)}\right] \\
B_{n}^{(4)}(t)=e^{-(\lambda+\mu+\gamma) t},
\end{gathered}
$$

where the notation $\delta_{i, j}$ is the Kronecker delta function and $\bar{G}(t) \stackrel{\text { def }}{=} 1-G(t)$. Let us substitute now into (2) and (4)-(7) the LT on the argument. Define

$$
b_{n}(s) \stackrel{\text { def }}{=} \int_{0}^{\infty} e^{-s t} B_{n}(t) d t, \quad \Re(s)>0,0 \leq n \leq N-1 .
$$

Since $b_{n}(s)=\sum_{i=1}^{4} \int_{0}^{\infty} e^{-s t} B_{n}^{(i)}(t) d t$ for $1 \leq n \leq N-1$, then we obtain now from (2) and (4)-(7) the following corresponding system of equations written for LTs:

$$
b_{0}(s)=\frac{1}{\lambda+s}\left(\lambda b_{1}(s)+1\right)
$$




$$
\begin{aligned}
b_{n}(s)=\frac{1}{\lambda+\mu+} & \gamma+s\left[\left(1-\delta_{n, N-1}\right) \lambda b_{n+1}(s)+\mu b_{n-1}(s)\right. \\
& \left.+\gamma \sum_{k=0}^{N-n-1}\left(g_{k}(s) b_{n+k}(s)+\bar{g}_{k}(s)\right)+1\right], \quad 1 \leq n \leq N-1,
\end{aligned}
$$

where we denote:

$$
\begin{aligned}
& g_{k}(s) \stackrel{\text { def }}{=} \frac{\lambda^{k}}{k !} \int_{0}^{\infty} e^{-(\lambda+s) t} t^{k} d G(t), \\
& \bar{g}_{k}(s) \stackrel{\text { def }}{=} \frac{\lambda^{k}}{k !} \int_{0}^{\infty} e^{-(\lambda+s) t} t^{k} \bar{G}(t) d t .
\end{aligned}
$$

\section{Compact-form solution for LT}

As one may notice, if we introduce the following sequences:

$$
\begin{gathered}
\alpha_{0}(s) \stackrel{\text { def }}{=} \frac{\mu}{\lambda+\mu+\gamma+s}, \\
\alpha_{n+1}(s) \stackrel{\text { def }}{=} \frac{1}{\lambda+\mu+\gamma+s}\left(\gamma g_{n}(s)+\delta_{n, 1}\left(1-\delta_{n, N-1}\right) \lambda\right), \quad n \geq 0, \\
\phi_{n}(s) \stackrel{\text { def }}{=}-\frac{1}{\lambda+\mu+\gamma+s}\left(\gamma \sum_{k=0}^{n-1} \bar{g}_{k}(s)+1\right), \quad 1 \leq n \leq N-1,
\end{gathered}
$$

the equations (10) can be transformed to the form:

$$
\sum_{k=-1}^{N-n-1} \alpha_{k+1}(s) b_{n+k}(s)-b_{n}(s)=\phi_{N-n}(s), \quad 1 \leq n \leq N-1 .
$$

The next step consists in using the substitution $\beta_{n}(s) \stackrel{\text { def }}{=} b_{N-n}(s)$. In consequence, we get from (9) and (15) the following system of equations:

$$
\begin{gathered}
\sum_{k=-1}^{n-1} \alpha_{k+1}(s) \beta_{n-k}(s)-\beta_{n}(s)=\phi_{n}(s), \quad 1 \leq n \leq N-1, \\
\beta_{N}(s)=\frac{1}{\lambda+s}\left(\lambda \beta_{N-1}(s)+1\right) .
\end{gathered}
$$

The infinite-sized system of equations (for $n \geq 1$ ) of type (16) was considered originally in [10]. It was proved there that its general solution can be represented in form:

$$
\beta_{n}(s)=M(s) R_{n}(s)+\sum_{k=1}^{n} R_{n-k}(s) \phi_{k}(s), \quad n \geq 1
$$

where successive terms of the sequence $R_{k}(s)$ can be computed via recursive formulae, namely 


$$
R_{0}(s) \stackrel{\text { def }}{=} 0, R_{1}(s) \stackrel{\text { def }}{=} \frac{1}{\alpha_{0}(s)}, R_{k+1}(s)=R_{1}(s)\left(R_{k}(s)-\sum_{i=0}^{k} \alpha_{i+1}(s) R_{k-i}(s)\right), k \geq 1 \text {. }
$$

Due to the fact that the number of equations in (16) is finite, then the correspondence (17) can be used as a specific-type boundary condition required for finding the formula for $M(s)$. Indeed, by taking $n=N$ in (18), we obtain

$$
\beta_{N}(s)=M(s) R_{N}(s)+\sum_{k=1}^{N} R_{N-k}(s) \phi_{k}(s) .
$$

Similarly, introducing (18) into (17), we get

$$
\beta_{N}(s)=\frac{1}{\lambda+s}\left[\lambda\left(M(s) R_{N-1}(s)+\sum_{k=1}^{N-1} R_{N-1-k}(s) \phi_{k}(s)\right)+1\right] .
$$

By comparing now the right sides of (20) and (21), we eliminate $M(s)$ as follows:

$$
M(s)=\frac{\sum_{k=1}^{N-1}\left(\frac{\lambda}{\lambda+s} R_{N-1-k}(s)-R_{N-k}(s)\right) \phi_{k}(s)+\frac{1}{\lambda+s}}{R_{N}(s)-\frac{\lambda}{\lambda+s} R_{N-1}(s)} .
$$

Now, by having in mind the relationship $\beta_{n}(s)=b_{N-n}(s)$ and by referring to (18) and (21), we can formulate the following main result:

Theorem 1: The LT $b_{n}(s)$ of the time-to-buffer overflow CDF tail, conditioned by the initial buffer state $n$, in the considered model of a single-machine manufacturing system with machine failures, can be written in the following form:

$$
\begin{array}{cl}
b_{n}(s)=\frac{R_{N-n}(s)\left[\sum_{k=1}^{N-1}\left(\frac{\lambda}{\lambda+s} R_{N-1-k}(s)-R_{N-k}(s)\right) \phi_{k}(s)+\frac{1}{\lambda+s}\right]}{R_{N}(s)-\frac{\lambda}{\lambda+s} R_{N-1}(s)} \\
+\sum_{k=1}^{N-n} R_{N-n-k}(s) \phi_{k}(s), & 0 \leq n \leq N-1,
\end{array}
$$

where the representations for $\phi_{k}(s)$ and $R_{k}(s)$ are given in (14) and (19), respectively.

Since for a non-negative random variable $X$ we have $\boldsymbol{E} X=\int_{0}^{\infty} \boldsymbol{P}\{X>x\} d x$, then from Theorem 1 the following corollary follows:

Corollary 1. The mean time to the buffer overflow $\boldsymbol{E T}_{n}$ in the considered manufacturing model, conditioned by the number $0 \leq n \leq N-1$ of jobs accumulated in the magazine before the starting epoch, is the following:

$$
\begin{aligned}
E T_{n}=b_{n}(0) & =\frac{R_{N-n}(0)\left[\sum_{k=1}^{N-1}\left(R_{N-1-k}(0)-R_{N-k}(0)\right) \phi_{k}(0)+\frac{1}{\lambda}\right]}{R_{N}(0)-R_{N-1}(0)} \\
& +\sum_{k=1}^{N-n} R_{N-n-k}(0) \phi_{k}(0) .
\end{aligned}
$$




\section{Numerical example}

As an illustrative example, let us consider the system described by the following parameters: $\lambda=\mu=4$ jobs/minute (equal arrival and departure rates), $\gamma=1$ (mean failurefree time equals 1 minute), exponentially distributed repairing times with mean 0.2 minute and the system size $N=6$. Three different initial levels of buffer saturation: empty buffer $(n=0)$, half-filled buffer $(n=3)$ and almost saturated buffer $(n=5)$ are considered in Figure 1, where transient evolution of conditional time-to-buffer overflow CDF tail is visualised. Moreover, average durations of time to buffer overflow equals, respectively: $3.985 ; 2.690$ and 1.050 .

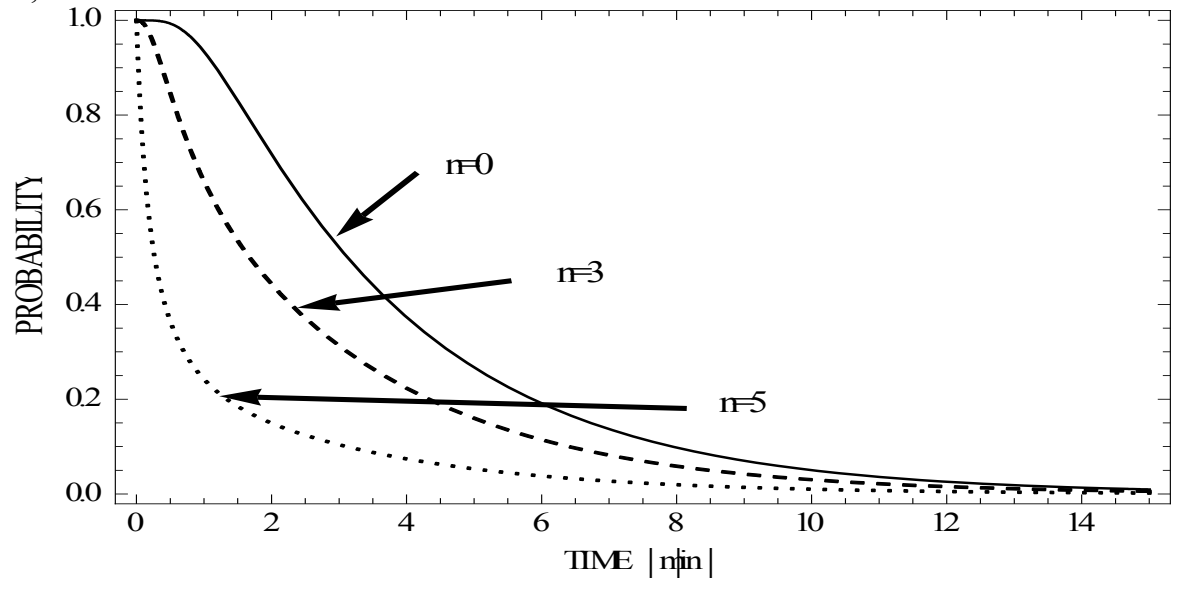

Fig. 1. Transient evolutions of time-to-buffer overflow conditional CDF tails.

\section{Conclusions}

A Markovian finite-buffer queue with machine breakdowns is investigated as a model of a single-channel manufacturing system. The compact-form formula for the Laplace transform of the time to the first buffer overflow conditional distribution is derived, by using the approach based on the paradigm of embedded Markov chain, total probability law and linear algebra. A numerical illustrative example is presented. The extension of the analysis is planned in future for the case of batch arrivals of jobs.

\section{References}

1. M.K. Govil, M.C. Fu, J. Manuf. Syst. 18 (3) (1999).

2. H.T. Papadopoulos, C. Heavey, Eur. J. Oper. Res. 92 (1) (1996).

3. A. Chydziński, Stoch. Models 23 (2007).

4. A. Chydziński, Lect. Notes Comput. Sci. 4479 (2007).

5. A. Al Hanbali, Queueing Syst. 67 (3) (2011).

6. E.Y. Lee, K.K.J. Kinateder, Stoch. Proc. Appl. 90 (1) (2000).

7. W.M. Kempa, I. Paprocka, Adv. Intel. Sys. 523 (2017).

8. W.M. Kempa, Proc. of 35th the International Conference Telecommunications and Signal Processing (TSP 2012), IEEE (2012).

9. W.M. Kempa, J. Adv. Telecommun. Electrotech. Signals Syst. 1 (2-3) (2012).

10. V.S. Korolyuk, Boundary-value problems for compound Poisson processes (Naukova Dumka, Kiev, 1975). 